Case Report

\title{
Spinal Subdural Hematoma in a Young Adult with Hemophilia A: A Case Report
}

\author{
Chunjiang Yan ${ }^{1,2}$, Ye Ye ${ }^{1,2,3,}$, Ruifeng Zeng ${ }^{1,2}$, Xiaoxin $\mathrm{Wu}^{1,2}$, Guorong Liang ${ }^{1,2}$, Huanmei $\mathrm{Li}^{1,2}$ \\ ${ }^{1}$ The Second Affiliated Hospital, Guangzhou University of Chinese Medicine, Guangzhou, China \\ ${ }^{2}$ Department of Emergency Medicine, Guangdong Provincial Hospital of Chinese Medicine, Guangzhou, China \\ ${ }^{3}$ Guangdong Provincial Key Laboratory of Research on Emergency in TCM, Guangzhou, China
}

Email address:

gdyeye@126.com (Ye Ye)

${ }^{*}$ Corresponding author

\section{To cite this article:}

Chunjiang Yan, Ye Ye, Ruifeng Zeng, Xiaoxin Wu, Guorong Liang, Huanmei Li. Spinal Subdural Hematoma in a Young Adult with Hemophilia A: A Case Report. Clinical Medicine Research. Vol. 9, No. 3, 2020, pp. 65-68. doi: 10.11648/j.cmr.20200903.14

Received: May 22, 2020; Accepted: June 12, 2020; Published: MM June 20, 2020

\begin{abstract}
Hemophilia is a set of hereditary hemorrhagic disorders of coagulation-dysfunction that can cause repeated bleeding in various tissues and organs. And the patient can be disabled due to bleeding from bones, joints, and soft tissue, or die due to splenic rupture or intracranial bleeding. So it is crucial to detect the site of bleeding in hemophilia patients quickly. Spontaneous spinal canal hematoma is a rare complication of hemophilia, with less than 50 cases reported to date. It can be easily missed and cause serious harm to patients. Hematoma, if untreated, causes irreversible nerve damage by compression of the spinal cord. Early recognition and treatment are most useful to prevent spontaneous spinal canal hematoma. We describe the case of a 21-year-old man with severe lumbago for two days, who had hemophilia A for a decade. The patient was diagnosed with spinal subdural hematoma by magnetic resonance imaging (MRI) after ruling out other causes of lumbago and treated with drugs by a neurologist. The hematoma showed absorption by MRI after six months. In conclusion, spontaneous spinal canal hematoma is a severe complication of hemophilia, which is often missed. This successful case confirms the importance of early diagnosis and treatment of spontaneous spinal canal hematoma. We hope to increase the clinicians' vigilance of this disease.
\end{abstract}

Keywords: Hemophilia, Spinal Subdural Hematoma, MRI, Rare Case, Case Report

\section{Introduction}

Hemophilia is a set of hereditary hemorrhagic disorders of coagulation-dysfunction. It can generally be divided into two types. Type A is the most common in factor VIII deficiency, and Type B in factor IX deficiency. Hemophilia can cause disability or death of patients. So it is vital to locate the bleeding site early.

Spinal epidural hematoma manifests bleeding in the epidural space along the spinal canal [1]. In 1869, a case of spinal epidural hematoma was first reported [2]. Few instances of spontaneous spinal canal hematoma have been reported [3, 4]. A subdural hematoma is rarer than epidural hematoma. Friday [5] reported a case of spontaneous spinal subdural hematoma with hemophilia and cerebral palsy, which is similar to this report.
While spontaneous spinal canal subdural or epidural hematoma is common, a subarachnoid hematoma is rare because it usually cannot form hematomas [6]. The incidence of spontaneous spinal canal epidural hematoma is about $0.1 / 100,000 /$ year [7] and accounts for $0.3-0.9 \%$ of spinal canal space-occupying lesion [8]. The etiology of spontaneous spinal epidural hematoma is trauma, including accidents and iatrogenic causes, such as an epidural catheter, blood coagulation dysfunction, anticoagulation, arteriovenous malformation, spinal canal hemangioma, Paget's disease [9, 10], but 40-50\% have unknown etiology [11]. The causes for the formation of subdural hematomas are similar to epidural hematomas. Coagulopathy and iatrogenic puncture of the vertebral canal generally leads to non-traumatic acute subdural hematoma of the intra-spinal canal [12]. 
Spontaneous epidural hematomas are easy to misdiagnose due to their obscure, diverse, or no apparent etiology, which could lead to permanent paralysis in the short-term. Specialized neurological knowledge and high vigilance are required for diagnosing spontaneous epidural hematomas.

The disease is characterized by insidious onset without foregleam, rapid development manifesting first as severe back pain consistent with spinal compression plane, quickly causing paralysis in a few hours or days. Paralysis is the end stage of the disease and portends poor prognosis [13]. Because of the importance of early diagnosis, timely magnetic resonance imaging (MRI) or computerized tomography (CT) should be performed for root pain, regardless of nerve dysfunction. MRI is more accurate than CT in diagnosing spontaneous epidural hematoma because it can accurately show the location and size of hematoma and the epidural [14]. MRI can clearly show spinal cord compression, since ventral subdural hematoma looks like a missing "curtain," during epidural hematoma in the dorsal spinal cord in the shape of a crescent moon. Acute hematoma in T1 weighted images showed equal or high signal, but the low signal in T2 weighted images. Features of subacute or chronic hematoma in T1 and T2 weighted images showed top sign with a clear boundary.

\section{Case Presentation}

The study was approved by the ethics committee of Guangdong Provincial Hospital of Chinese Medicine. Informed consent has been obtained. A 21-year-old man, whose brother, a hemophiliac, died of a cerebral hemorrhage at 16, was preliminarily diagnosed with hemophilia in 1997 because of subcutaneous hematoma in his thighs, neck, double arm, and knee joint swelling with pain accompanied by limited activity. Factor VIII treatment improved his symptoms. The examination showed F VIII 9.5\% C, FIX C 180\%, PT 10.8 $\mathrm{s}$, APTT $106.3 \mathrm{~s}$, and PLT $301 \mathrm{x} 10 \mathrm{e} 9$. He was finally diagnosed with hemophilia A in May 2004 and repeatedly infused with factor VIII and fresh frozen plasma for the subcutaneous hematoma and bloody urine. In 2012, his knees were swollen, and in mid-august, he developed backache. The lumbago was severe without radiating pain in lower limbs on August 21 and worsened in two days. He was unable to stand due to the swollen left leg and spastic lower back pain when He visited the Emergency Room on August 23. Physical examination showed mild tenderness and percussion pain over the kidney region and no pathological reflex. Labs: white blood cell (WBC) $12.73 * 10 \mathrm{E} 9 / \mathrm{L}$, activated partial thromboplastin time (APTT) $105.7 \mathrm{~s}$, and coagulation factor VIII activity $1 \%$. A plain abdominal radiograph showed pneumatosis in the colon and small intestine. Lumbar radiographs showed that the physiological curvature had become straight with no abnormal vertebral bone. The patient did not agree to the lumbar MRI examination and was kept under observation and treated with factor VIII 2000iu q $12 \mathrm{~h}$ infusion, which was not very useful. Labs showed factor VIII activity $25 \%$ on August 26 , after which the patient agreed to lumbar MRI on the same day. MRI showed abnormal signals in T12-S1 levels in the spinal canal, considered as hematoma, and the lumbar signal had generally decreased, which was consistent with the history of hemophilia [Figure 1]. The diagnosis of intraspinal subdural hematoma was finalized after orthopedics consultation. Tranexamic acid and sodium chloride injection, $100 \mathrm{ml}$ ivd $\mathrm{qd}$, and polyene phosphatidylcholine capsules, and $0.228 \mathrm{~g}$ PO tid, were added to the psychiatrist's therapeutic regimen. As the backache gradually improved, the patient was discharged on September 12. He was reviewed by lumbar MRI without backache symptom on April 24, 2013, which showed a decrease in lumbar signal, combined with a history of hemophilia and abnormal signals in L1-5 level of the spinal canal, conforming to chronic hematoma absorption [Figure 2].

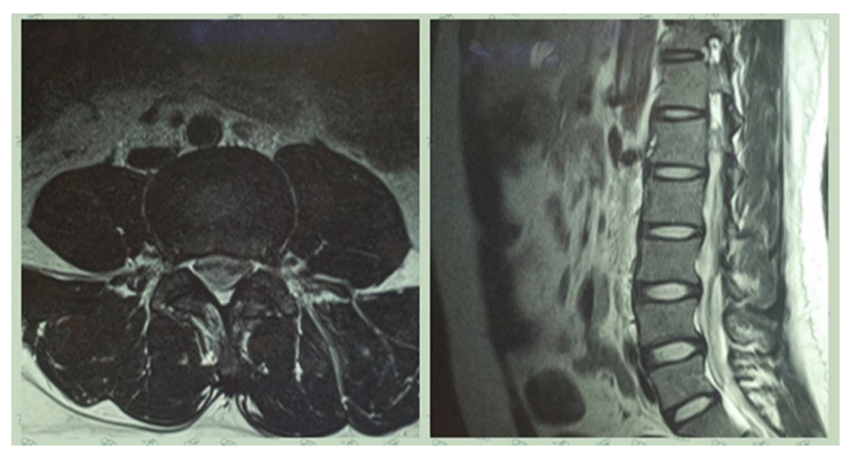

Figure 1. August 26, 2012 MRI.

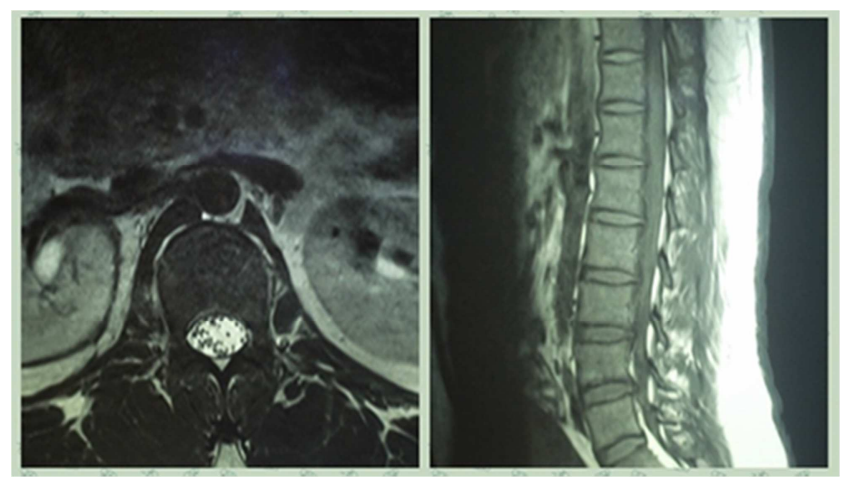

Figure 2. April 24, 2013 MRI without backache symptom.

\section{Discussion}

In this case, neurological complications attracted attention after excluding trauma, strain, infection, inflammation [15]. Based on physical examination and labs, because backache was the main symptom of the patient. Backache is the most common symptom of paresthesia when nerve cells are damaged from bodily injury, viruses, or drugs. Physical oppression causes the majority of back pain and can be clearly seen in the form of compression and compressed vertebrate by MRI, which is better than CT in discerning intraspinal soft tissue and understanding the anatomical characteristics of the spinal tissue in all planes. MRI can easily find a small hemorrhage and accurately reflect its size, shape, location, and relationship with the surrounding tissue. Moreover, tumors and inflammation can be distinguished by a signal difference 
in MRI [16]. In this case, MRI clearly indicated hematoma in the T12-S1 level spinal canal based on the abnormal signal.

Minor bleeding due to various causes can quickly stop in healthy people because the clotting mechanism rapidly takes effect. However, this is not the case in hemophiliacs [17]. The disorder between clotting and anticoagulant mechanism may cause non-stop bleeding. A large volume of venous blood collected in the epidural venous plexus increases the pressure when intra-abdominal pressure is increased by coughing, sneezing, or bearing down during bowel movements, which may cause rupturing of blood vessels. The hematoma will gradually form from oozing blood and grind against the spinal cord causing backache in hemophiliacs. Increased hematoma and compressed spinal cord are extremely likely to cause paraplegia.

Our patient's coagulative function was so poor that the operation was thought to be too risky after consultation with neurologist [18]. Therefore, tranexamic acid was given to stop bleeding and polyene phosphatidylcholine for the trophic nerve. The patient felt improvement in backache without abnormal sensation and movement disorders of lower limbs after a week. We found no increase in the hematoma and discharged the patient with drug treatment. An MRI review after seven months showed that the hematoma had reduced and confirmed our conjecture.

The probability of paraplegia in this patient was overwhelming because backache was exacerbating, although numbness, weakness, and other sensory movement disorders did not appear, suggesting that the hematoma was increasing, and the nerve cells were damaged. A timely MRI examination and effective medication cured this patient. This case indicates that imaging examination should be conducted timely in hemophiliacs and other coagulopathy patients with the backache.

\section{Conclusions}

Spontaneous spinal canal hematoma is a severe complication of hemophilia that is often missed. In this case, timely MRI showed spinal nerve compression due to hematoma that ranged from $\mathrm{T} 12$ to $\mathrm{S} 1$ level of the spinal canal. It would have caused irreversible neurological damage if not managed with clotting factors, hemostatic, and trophic nerve. Minimally invasive surgery can also reduce hematoma. This case corroborates the importance of early diagnosis and treatment of spontaneous spinal hematoma. We hope to increase the vigilance of clinicians about this disease through this case report.

\section{Conflict of Interest}

The authors declare that they have no competing interests.

\section{Acknowledgements}

This work was supported by the Guangdong Provincial Key Laboratory of Research on Emergency in TCM (2017B030314176). The funders of this study have no role in any activities or decisions relating to study design, data collection, data management, data analysis, interpretation of data, report writing, and report publication.

\section{References}

[1] Lillemäe K, Järviö JA, Silvasti-Lundell MK, Antinheimo JJ, Hernesniemi JA, Niemi TT. Incidence of Postoperative Hematomas Requiring Surgical Treatment in Neurosurgery: A Retrospective Observational Study. World Neurosurg. 2017; 108: 491-497.

[2] Lawton MT, Porter RW, Heiserman JE, Jacobowitz R, Sonntag VK, Dickman CA. Surgical management of spinal epidural hematoma: relationship between surgical timing and neurological outcome. J Neurosurg 1995; 83: 1-7.

[3] Groen RJ, Van Alphen HA. Operative treatment of spontaneous spinal epidural hematomas: a study of the factors determining postoperative outcome. Neurosurgery 1996, 39: 494-508; discussion 508-499.

[4] Huang. G. F, Liu. D. H. Epidural hematoma of spinal cord. Foreign Medical Sciences Section on Neurology \& Neurosurgery 1996; 23: 169-172.

[5] Friday RY, Pollack IF, Bowen A, Pollack A, Ragni M. Spontaneous spinal subdural hematoma in a young adult with hemophilia. J Natl Med Assoc 1999; 91: 289-294.

[6] Cabrera Manchola A, Manchola I, Larena JA, Martinez de Guerenu B, Casado O. Follow up of a spontaneous ventral subarachnoid spinal haematoma by means of magnetic resonance. Rev Neurol 2001; 32: 1137-1140.

[7] Holtas S, Heiling M, Lonntoft M. Spontaneous spinal epidural hematoma: findings at MR imaging and clinical correlation. Radiology 1996; 199: 409-413.

[8] Oldenkott P, Preger R, Todorow S. Spinal epidural hematoma and anticoagulation treatment. Med Welt 1981; 32: 46-49.

[9] Domenicucci M, Ramieri A, Ciappetta P, Delfini R. Nontraumatic acute spinal subdural hematoma: report of five cases and review of the literature. J Neurosurg 1999; 91: 65-7.

[10] Park JH, Park S, Choi SA. Incidence and risk factors of spinal epidural hemorrhage after spine surgery: a cross-sectional retrospective analysis of a national database. BMC Musculoskelet Disord. 2020; 21 (1): 324.

[11] Kobayashi K, Ando K, Nishida Y, Ishiguro N, Imagama S. Epidemiological trends in spine surgery over 10 years in a multicenter database. Eur Spine J. 2018; 27 (8): 1698-1703.

[12] Oichi Y, Toda H, Yamagishi K, Tsujimoto Y. Multiple Spinal Chronic Subdural Hematomas Associated with Thoracic Hematomyelia: A Case Report and Literature Review. World Neurosurg. 2019; 131: 95-103.

[13] Masuda S, Fujibayashi S, Takemoto M, et al. Incidence and Clinical Features of Postoperative Symptomatic Hematoma after Spine Surgery: A Multicenter Study of 45 Patients. Spine Surg Relat Res. 2019; 4 (2): 130-134.

[14] Tavolaro C, Ghaffar S, Zhou H, Nguyen QT, Bellabarba C, Bransford RJ. Is routine MRI of the spine necessary in trauma patients with ankylosing spinal disorders or is a CT scan sufficient? Spine J. 2019; 19 (8): 1331-1339. 
[15] Lagerkranser M, Lindquist C. Neuraxial blocks and spinal haematoma: Review of 166 cases published 1994 - 2015. Part 2: diagnosis, treatment, and outcome. Scand J Pain. 2017; 15: 130-136.

[16] Maddali P, Walker B, Fisahn C, et al. Subdural Thoracolumbar Spine Hematoma after Spinal Anesthesia: A Rare Occurrence and Literature Review of Spinal Hematomas after Spinal Anesthesia. Cureus. 2017; 9 (2): e1032.
[17] Hegde A, Nair R, Upadhyaya S. Spontaneous intracerebral hemorrhage in hemophiliacs-A treatment dilemma. Int J Surg Case Rep. 2016; 29: 17-19.

[18] Shalabi A, Kachel E, Kogan A, et al. Cardiac surgery in patients with Hemophilia: is it safe?. J Cardiothorac Surg. 2020; 15 (1): 76. 